\title{
Prevalence of minimal hepatic encephalopathy among patients with chronic liver disease in Ilorin, Nigeria
}

\author{
Opeyemi F. Bamidele ${ }^{1}$, Abdulfatai B Olokoba ${ }^{1}$, Matthew O Bojuwoye ${ }^{1}$, Richard O Akintayo ${ }^{1}$ and \\ Oluwakemi Bamidele ${ }^{2}$
}

Ghana Med J 2019; 53(4): 299-303 doi: http://dx.doi.org/10.4314/gmj.v53i4.8

\author{
${ }^{1}$ Department of Medicine, University of Ilorin Teaching Hospital, Ilorin. Nigeria \\ ${ }^{2}$ Department of Behavioural Sciences, University of Ilorin Teaching Hospital, Ilorin. Nigeria
}

\author{
Corresponding author: Opeyemi F. Bamidele $\quad$ E-mail: Opebamidele097@gmail.com \\ Conflict of interest: None declared
}

\section{SUMMARY}

Background: Minimal Hepatic encephalopathy is the mildest form of Hepatic Encephalopathy which presents with significant cognitive impairment and affectation of activities of daily living. The literature is scanty on the prevalence of minimal hepatic encephalopathy in Nigerians with chronic liver disease.

Aim: This study aimed at determining the prevalence of minimal hepatic encephalopathy among patients with chronic liver disease using neuro-psychometric tests.

Methods: The study was a hospital-based cross-sectional study carried out at the University of Ilorin Teaching Hospital, Ilorin from February 2015 to February 2016. Chronic liver disease was diagnosed with the presence of peripheral stigmata of liver disease, liver biochemistry, prothrombin time, and sonographic findings in keeping with liver disease. Minimal hepatic encephalopathy was diagnosed using number connection tests-A and B for patients who were educated while Line tracing test and constructional dyspraxia were used for patients without any formal education. Data obtained were analysed using Statistical Package for the Social Sciences (SPSS) version 20 computer software package.

Results: Sixty-four patients with chronic liver disease were recruited. The mean age (SD) of the patients was $47.1 \pm 14.6 \mathrm{yrs}$, and the $30-39$ and $40-49$ yrs age groups each had the highest frequency of $21(32.8 \%)$. There were 54 (84.4\%) males and 10 (15.6\%) females. The prevalence of Minimal Hepatic Encephalopathy was 43.8\%.

Conclusion: The prevalence of Minimal Hepatic Encephalopathy in this study was similar to previous studies. Significant number of patients with minimal HE were in Child-Pugh class B and C.

Keywords: Minimal, Hepatic Encephalopathy, Liver Disease, Neuro-psychometric test Funding: None

\section{INTRODUCTION}

Hepatic encephalopathy (HE) is a neuropsychiatric manifestation of a failing liver which is potentially reversible with appropriate intervention and is a complication of portal hypertension with an associated porto-systemic shunt. ${ }^{1} \mathrm{HE}$ has been extensively studied in the past, and it has been classified into HE associated with acute liver failure (Type A), HE associated with liver by-pass without intrinsic liver disease (Type B) and HE associated with liver cirrhosis (Type C). Type C HE can be further sub-classified based on time course (episodic, persistent) and grade (minimal HE and overt HE). ${ }^{2}$ Minimal hepatic encephalopathy (MHE) is the mildest form of HE which presents with significant cognitive impairment and affectation of activities of daily living. ${ }^{3}$
MHE is defined as HE without symptoms/signs on clini$\mathrm{cal} /$ neurological examination, but with impairment in some cognitive areas that can only be determined by neuro-psychometric testing. ${ }^{2}$ The prevalence of MHE is said to be high among patients with liver cirrhosis and even higher in the presence of poor liver function. It is reported to vary between $30-84 \% .^{3,4}$ Although there have been arguments about the exact pathogenetic mechanism of MHE, certain factors such as hyper-ammonia and increased blood-brain permeability to ammonia, elevated brain concentration of manganese, inflammation and inhibitory neurosteroids have been hypothesised in the pathogenesis of MHE. ${ }^{5}$ 


\section{Original Article}

At the same time several factors such as sepsis, gastrointestinal bleeding, constipation, uraemia, hypokalae$\mathrm{mia} /$ hyponatraemia, diuretics, sedatives, etc, have been known to alter the thin balance of liver function in cirrhosis thereby precipitating or worsening pre-existing HE.

Minimal hepatic encephalopathy as the mildest form in the spectrum of HE is difficult to diagnose due to its subclinical nature and also due to lack of consensus on which diagnostic tool to use. ${ }^{6}$ The diagnosis of MHE requires an impairment in performance of at least two of the neuro-psychometric tests in the absence of an abnormal neurological examination, and also of features of overt HE. $^{2}$ The criteria for diagnosis of HE is impairment in at least two of neuro-psychometric tests, that is two standard deviations (2SD) below age- and education-matched healthy control. ${ }^{2}$

The burden of MHE is enormous with clinical and social impact on the patients as they are frequently undiagnosed. These patients tend to have a poor quality of life in the aspect of sleep, alertness, and functional capacity. ${ }^{7}$, ${ }^{8}$ Complex activities as attention, information processing and psychomotor skills such as driving a car, planning a trip are mainly affected. ${ }^{9}$ It was found that patients with MHE had impaired performance in tests of short and long term memory that require free recall or recognition. ${ }^{10}$ MHE is also associated with increased risk of developing overt HE and overall mortality. ${ }^{11}$

There is paucity of data on the prevalence of MHE in Nigeria despite its substantial negative effect on the quality of life, hence the need to identify MHE as it also predicts overt HE. This study aimed to determine the prevalence of MHE among patients with chronic liver disease (Liver cirrhosis and Hepatocellular carcinoma) in University of Ilorin Teaching Hospital (UITH) using neuro-psychometric test as the diagnostic tool.

\section{METHODS}

The study was a hospital-based cross-sectional study. It was carried out at UITH, Ilorin, Nigeria. All patients with Chronic Liver Disease (CLD) attending the Gastroenterology clinic, and all in-patients admitted for CLD were recruited into the study from February 2015 to February 2016. A patient was recruited if he/she had a background CLD based on typical clinical findings, liver biochemistry with or without sero-positivity for Hepatitis B and C virus, and sonographic findings in keeping with liver cirrhosis or hepatocellular carcinoma (HCC). Hepatocellular carcinoma was diagnosed using elevated serum alpha feto-protein levels ( $\geq 400 \mathrm{ng} / \mathrm{ml})$ with a typical abdominal ultrasound scan and/or abdominal computer tomography findings suggestive of $\mathrm{HCC} .^{12}$
A diagnosis of MHE was made if there was a normal neurological examination and impairment in the performance of both number connection test A (NCT-A) and number connection test $\mathrm{B}$ (NCT-B), i.e. patients used more than 2SD of the mean time in completing NCT-A and NCT-B each for healthy subjects. ${ }^{2}$ Therefore, patients who used more than 93 seconds and 188 seconds for completing NCT-A and NCT-B respectively were considered to have MHE.

Among patients without formal education, MHE was diagnosed if there was a normal neurological examination but patients used more than the 2SD of mean time in completing the line tracing test (LTT) for healthy subjects (i.e. a value greater than 240 seconds) and also in the presence of constructional dyspraxia (inability to construct a cube with a match stick or draw a five-pointed star). ${ }^{2}$ Patients without clinical, laboratory nor sonographic evidence of liver cirrhosis and/or HCC were excluded from the study. Patients with overt HE were also excluded from the study.

Patients who had symptoms suggestive of upper gastrointestinal bleeding such as haematemesis and melaena had upper gastro-intestinal endoscopy to identify the source of bleed and endoscopic therapy. Diagnosis of spontaneous bacterial peritonitis was based on an ascitic neutrophil count of at least $250 \mathrm{cells} / \mathrm{mm}^{3}$, with or without a positive ascitic fluid culture. ${ }^{13}$ Serum sodium, potassium, creatinine and urea were determined to detect any derangement that could be a potential precipitant of MHE.

To identify infection and its focus, a complete blood count, blood culture, urinalysis was done for all patients, while urine microscopy, culture and sensitivity (mcs) was done for patients with urinary symptoms, and sputum mcs for those with productive cough. Other precipitants of HE, such as constipation (bowel movement less than three times in a week), and the use of sedatives were sought for among patients. The NCT-A, NCT-B and LTT were standardized using 64 apparently healthy subjects, who were matched with the CLD patients for age, sex and educational status.

They were selected from the staff of the hospital and relatives of the patients using a convenient method of sampling. They were all negative for Hepatitis B surface Antigen and anti-Hepatitis C Virus and had normal liver biochemistry and abdominal sonography.

A standardised, semi-structured questionnaire was used to extract the biodata, information on clinical features of CLD and laboratory findings from each patient. 
Brain imaging with computerized tomographic scan was done where possible to exclude primary neurological cause or where a diagnosis of HE was in doubt. The Child-Pugh scoring system was used for assessing the severity of liver disease using clinical and laboratory parameters. ${ }^{14,15}$ The scoring system takes into account the serum albumin, prothrombin time, and bilirubin as well as the presence of ascites and encephalopathy; each of which is given a numerical score and subsequently graded as A, B, and C depending on the total scores.

Ethical approval was obtained from the Ethics and Research committee of UITH (UITH/CAT/189/18 $/ 917$ )

\section{RESULTS}

Sixty-four patients were studied comprising, 43 (67.2\%) out-patients and 21 (32.8\%) in-patients. Patients with features of overt HE were excluded from data analysis. The age of the patients recruited for the study ranged from 20 to 90 years with a mean age of $47.1 \pm 14.6$ years. The highest number of patients was within the age range of 30-49 years (65.6\%). More than three-quarters of the patients studied were males (84.4\%) with a female to male ratio of 1:4.8. Majority of the patients had a form of formal education $(70.3 \%)$ while the remaining $(29.7 \%)$ patients had no formal education (Table 1).

Table 1 Socio-demographic characteristics of patients with CLD

\begin{tabular}{|l|l|}
\hline Age group & $\begin{array}{l}\text { Frequency } \\
\text { n (\%) }\end{array}$ \\
\hline $20-29$ & $4(6.3)$ \\
\hline $30-39$ & $21(32.8)$ \\
\hline $40-49$ & $21(32.8)$ \\
\hline $50-59$ & $8(12.5)$ \\
\hline $60-69$ & $5(7.8)$ \\
\hline $70-79$ & $3(4.7)$ \\
\hline $80-90$ & $2(3.1)$ \\
\hline Gender & \\
\hline Male & $54(84.4)$ \\
\hline Female & $10(15.6)$ \\
\hline Level of education & \\
\hline None & $19(29.7)$ \\
\hline Primary & $7(10.9)$ \\
\hline secondary & $11(17.2)$ \\
\hline Post-secondary & $27(42.2)$ \\
\hline
\end{tabular}

A total of $28(43.8 \%)$ patients had MHE. NCT-A and NCT-B were used for CLD patients with formal education, and 17 patients spent more than 93 seconds for NCT-A and more than 188 seconds for NCT-B. Among the patients without formal education, 11 individuals spent more than 240 seconds to complete the LTT and also had constructional dyspraxia (Table 2).
Table 2 Results of neuro-psychometric test of patients with chronic liver disease

\begin{tabular}{|c|c|c|c|}
\hline $\begin{array}{l}\text { Time in connecting num- } \\
\text { bers for patients with formal } \\
\text { education }\end{array}$ & & $\begin{array}{l}\text { No MHE } \\
\text { N (\%) }\end{array}$ & $\begin{array}{l}\text { MHE } \\
\text { N (\%) }\end{array}$ \\
\hline \multirow[t]{2}{*}{ NCT-A } & $<93$ seconds & $28(43.8)$ & - \\
\hline & $>93$ seconds & - & $17(26.6)$ \\
\hline \multirow[t]{2}{*}{ NCT-B } & $<188$ seconds & $28(43.8)$ & - \\
\hline & $>188$ seconds & - & $17(26.6)$ \\
\hline Constructional apraxia & & - & $17(26.6 \%)$ \\
\hline \multicolumn{4}{|c|}{ Time in tracing line for patients without Formal education } \\
\hline & $<240$ seconds & $8(12.5)$ & - \\
\hline & $>240$ seconds & - & $11(17.2)$ \\
\hline \multicolumn{2}{|c|}{ Patients with constructional apraxia } & $8(12.5)$ & $11(17.2)$ \\
\hline
\end{tabular}

The majority of patients with MHE were males (78.6\%) within 30 to 49 years of age. Most (64.3\%) of them had formal education (Table 3).

Table 3 Distribution of Hepatic Encephalopathy by socio-demographic characteristics

\begin{tabular}{|l|l|c|}
\hline Variable & NO HE & MHE \\
\hline Age groups & N (\%) & N (\%) \\
\hline $\mathbf{2 0}$-29 & $2(8.1)$ & $1(3.6)$ \\
\hline $\mathbf{3 0 - 3 9}$ & $15(40.5)$ & $7(25.0)$ \\
\hline $\mathbf{4 0 - 4 9}$ & $14(37.9)$ & $7(25.0)$ \\
\hline $\mathbf{5 0}-\mathbf{5 9}$ & $3(8.1)$ & $5(17.9)$ \\
\hline $\mathbf{6 0}-\mathbf{6 9}$ & $1(2.7)$ & $4(14.3)$ \\
\hline $\mathbf{7 0}-\mathbf{7 9}$ & $1(2.7)$ & $2(7.1)$ \\
\hline $\mathbf{8 0 - 9 0}$ & - & $2(7.1)$ \\
\hline & & \\
\hline Gender & & $22(78.6)$ \\
\hline Male & $32(89.2)$ & $6(21.4)$ \\
\hline Female & $4(10.8)$ & \\
\hline & & $10(35.7)$ \\
\hline Level of education & & $1(3.6)$ \\
\hline No formal education & $9(24.3)$ & $4(14.3)$ \\
\hline Primary & $6(16.2)$ & $13(46.4)$ \\
\hline Secondary & $7(19.0)$ & \\
\hline Tertiary & $14(40.5)$ & \\
\hline
\end{tabular}

A total of 23 (63.9\%) patients among those without MHE were in Child-Pugh class $\mathrm{B}$ or $\mathrm{C}$ while among patients with MHE, 23 (82.1\%) were in Child-Pugh class B or C (Figure 1).

The following were some of the known precipitants identified in patients with MHE, Gastro-intestinal bleeding (8), hypokalaemia (7), Spontaneous bacterial peritonitis (4), other infections (11), constipation (6), patients with unidentified precipitants were two. 


\section{Original Article}

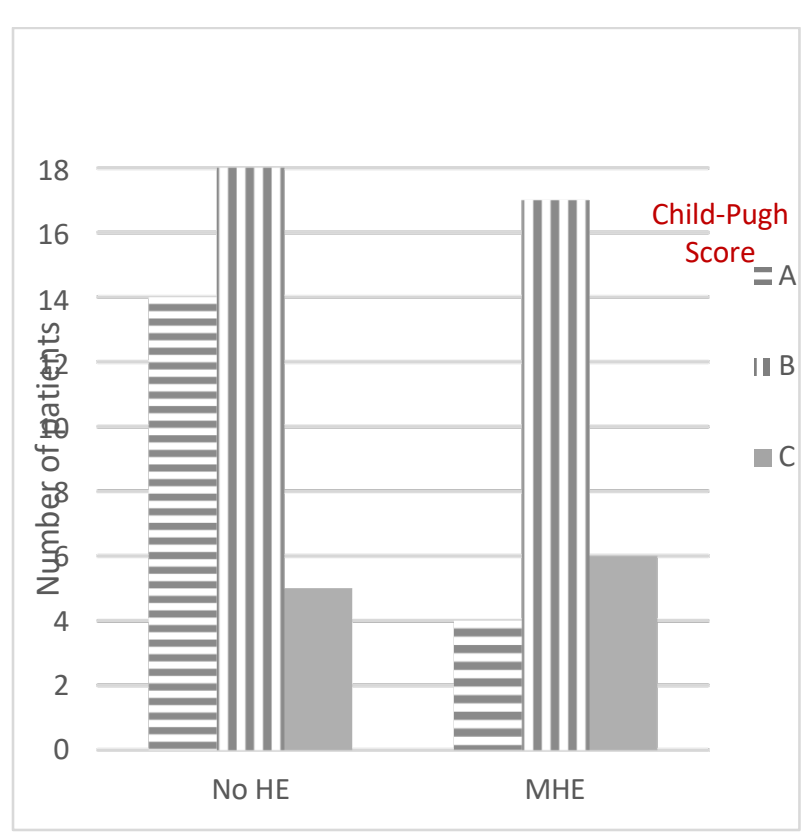

Figure 1 Child-Pugh classification among patients with and without MHE

\section{DISCUSSION}

Hepatic encephalopathy is an important neuropsychiatric complication of liver disease. The prevalence of MHE among CLD patients differ from one locality to another as do the various risk factors/ aetiology of CLD.

The mean age of the patients in this study contrasts with that by Karen et al in the United Kingdom, where a mean of 57.0 \pm 7.0 years was reported. ${ }^{16}$ Groeneweg et al also stated that CLD patients who develop MHE were elderly. $^{17}$

However, Olokoba et al reported an average age of 32.9 years in their study. ${ }^{18}$ The majority of our patients with MHE were between the ages of 30 to 49 years; this was not surprising as most of the patients with CLD in our study were within this age bracket. The dominant middle age group in this study can be explained by the endemicity of HBV infection in this part of the world and also the predominant horizontal transmission of HBV infection in the perinatal period and early childhood. Hence many of these patients develop HBV-induced CLD early in life. To buttress this statement, over three-quarter of the patient with CLD in our study had Hepatitis B infection $(78 \%)$.

The male predominance in this study may be due to the fact that males are more exposed to the risk factors predisposing to CLD such as alcohol intake, ingestion of herbal concoction, multiple sexual partners and sharing of sharps. In addition, Yu et al demonstrated that the male sex hormone testosterone was significantly higher in
HBsAg-positive HCC when compared with controls. ${ }^{19}$ In our study, more than half of the CLD patients had a formal education, and this pattern was also seen among patients with MHE.

Olokoba et al in a study on the risk practices towards liver disease in Ilorin observed that irrespective of the educational status of patients there was a low level of awareness among the study population with a poor perception of the risk factors for liver disease and a misconception of not being at personal risk of liver disease. ${ }^{18}$ This observation may explain the fact that despite the high level of education among the study population, there may be a similar poor perception with regards to the risk factors, nature and degree of severity of CLD. Furthermore, illiterates are more likely to be involved in risk practices towards CLD such as scarification, local circumcision, and ingestion of herbal concoction and also tend to have poor health-seeking behaviour.

In this study, the prevalence of MHE was $43.8 \%$, a percentage similar to the findings by Nwabuaku et $a l^{20}$ in Lagos, south west Nigeria, who reported a prevalence of 43.1\%. However, an abnormal electro-encephalography (EEG) was added to the diagnostic criteria in their study. The prevalence of MHE in this study was comparable to the $41.0 \%$ reported by Praveen et al, ${ }^{21}$ in Saudi Arabia, where more sophisticated diagnostic tools such as P300 auditory event potential (P300ERP) and critical flicker frequency (CFF) with psychometry were used to diagnose MHE. The prevalence of MHE in this study was higher than the $32.7 \%$ reported by Maldonado-Garja et $a l^{22}$ in Mexico, and $34.0 \%$ reported by Barbosa et $a l^{23}$ in Portugal.

The differences observed may be attributed to the different diagnostic tests used in detecting $\mathrm{HE}$ in each study, differences in the time the studies were conducted, and probable geographical differences. The high prevalence of MHE in our study could also be attributed to the advance liver disease at presentation, as the majority of these patients were either in Child-Pugh class B or C. The proportion of patients with liver cirrhosis who had MHE in our study $(53.6 \%)$ were comparable to previous studies. $^{3,4,11}$ The occurrence of MHE increases with increasing Child-Pugh score. Among patients with MHE, it was observed that many of them were in Child-Pugh class B and $\mathrm{C}$, only a few patients with MHE were in Child-Pugh class A. To buttress these findings, Praveen et al in Saudi Arabia, ${ }^{21}$ reported that $36 \%$ and $56 \%$ of patients with MHE were in Child-Pugh score grade $\mathrm{B}$ and $\mathrm{C}$ respectively. 
As there is no consensus on the diagnosis of MHE, it will be worth recommending the use of neuro-psychometric tests specifically NCT and LTT in diagnosing MHE as they cover the cognitive aspects that are affected in MHE. They are also cheap, reproducible and accessible.

Limitation in this study was that sophisticated tools used in diagnosing MHE such as CFF and P300ERP were not used as these tools were unavailable as at the time of this study.

\section{CONCLUSION}

The prevalence rate of MHE obtained in this study is similar to rates obtained from previous studies including studies that combined psychometric tests with other neurophysiological tests such as EEG, Critical flicker frequency. A significant number of patients with MHE were in Child-Pugh class B and C.

\section{REFERENCES}

1. Blei AT, Córdoba J. Hepatic encephalopathy. Am J Gastroenterol. 2001;96(7):1968.

2. Ferenci P, Lockwood A, Mullen K, Tarter R, Weissenborn K, Blei AT. Hepatic encephalopathy--definition, nomenclature, diagnosis, and quantification: Final report of the working party at the 11th world congresses of Gastroenterology, Vienna, 1998. Hepatol. 2002;35(3):716-21.

3. Dhiman RK, Chawla YK. Minimal hepatic encephalopathy. Indian J Gastroenterol. 2009;28(1):5-16.

4. Das A, Dhiman RK, Saraswat VA, Verma M, Naik SR. Prevalence and natural history of subclinical hepatic encephalopathy in cirrhosis. $J$ Gastroenterol hepatol. 2001;16(5):531-5.

5. Bleibel W, Al-Osaimi A. Hepatic encephalopathy. Saudi J Gastroenterol. 2012;18(5):301-9.

6. Bajaj JS, Etemadian A, Hafeezullah M, Saeian K. Testing for minimal hepatic encephalopathy in the united states: An aasld survey. Hepatology. 2007;45(3):833-4.

7. Schomerus H, Hamster W. Quality of life in cirrhotics with minimal hepatic encephalopathy. Metab Brain Dis. 2001;16(1-2):37-41.

8. Groeneweg M, Quero JC, De Bruijn I, Hartmann IJ, Essink-bot ML, Hop WC, et al. Subclinical hepatic encephalopathy impairs daily functioning. Hepatology. 1998;28(1):45-9.

9. Arguedas MR, DeLawrence TG, McGuire BM. Influence of hepatic encephalopathy on health-related quality of life in patients with cirrhosis. Dig dis sci. 2003;48(8):1622-6.

10. Weissenborn K, Heidenreich S, Giewekemeyer K, Ruckert N, Hecker H. Memory function in early hepatic encephalopathy. J hepatol. 2003;39(3):320-5.
11. Dhiman RK, Kurmi R, Thumburu KK, Venkataramarao SH, Agarwal R, Duseja A, et al. Diagnosis and prognostic significance of minimal hepatic encephalopathy in patients with cirrhosis of liver. Dig dis sci. 2010;55(8):2381-90.

12. Ryder SD. Guidelines for the diagnosis and treatment of hepatocellular carcinoma (HCC) in adults. Gut 2003; 52:1-8

13. Runyon BA. Ascites and spontaneous bacterial peritonitis. In; Feldman M, Friedman LS, Sleisenger $\mathrm{MH}$, eds. Sleisenger and Fordrans gastrointestinal and liver disease,8th ed. Philadephia: Saunders,2006: 1935-1964.

14. Cholongitas E, Papatheodoridis GV, Vangeli M, Terreni N, Patch D, Burroughs AK. "Systematic review: The model for end-stage liver disease--should it replace Child-Pugh's classification for assessing prognosis in cirrhosis?". Alimentary Pharmacology \& Therapeutics. 2005; 22 (11-12): 1079-89

15. Child CG, Turcotte JG. "Surgery and portal hypertension". In Child CG. The Liver and portal hypertension. 1964; Philadelphia: Saunders. pp. 50-64.

16. Thomsen KL, Macnaughtan J, Tritto G, Mookerjee RP, Jalan R. Clinical and pathophysiological characteristics of cirrhotic patients with grade 1 and minimal hepatic encephalopathy. PloS one. 2016;11(1):e0146076.

17. Groeneweg M, Moerland W, Quero JC, Hop WC, Krabbe PF, Schalm SW. Screening of subclinical hepatic encephalopathy. J hepatol. 2000;32(5):748-53.

18. Olokoba AB, Aderibigbe S, Kayode OO. A community survey of awareness and risk perception for liver diseases among adults in Ilorin metropolis. Am J Sci Ind Res.2010. 109-15

19. Yu M-W, Yang Y-C, Yang S-Y, Cheng S-W, Liaw Y-F, Lin S-M, et al. Hormonal markers and hepatitis $\mathrm{b}$ virus-related hepatocellular carcinoma risk: A nested case-control study among men. J Natl Cancer Inst .2001;93(21):1644-51.

20. Nwabuaku O, Onyekwere C, Ogun S. Minimal hepatic encephalopathy amongst Chronic liver disease patients in a hospital: Need for cognitive evaluation? Nig. J. Gastroenterol. Hepatol . 2016;8(1):25-33.

21. Praveen S, Bargesh CS. Predictors of minimal hepatic encephalopathy in patients with cirrhosis. Saudi J Gastroenterol 2010; 16:181-187.

22. Maldonado-Garza HJ, Varquez-Elizondo G, Gaytan-Torres JO, Flores-Rendon AR, Cardenas-Sandoval MG, Bosques-Padilla FJ. Prevalence of minimal hepatic Encephalopathy in Cirrhotic Patients. Ann Hepatol .2011; 10: 40-44.

23. Barbosa M, Marinho C, Mota P, Cotter J. Minimal hepatic encephalopathy: The reality Beyond our eyes. Acta Medica Portuguesa. 2015;28 (4):480-5 